\title{
Compatible Changes of Lead microRNAs in Circulating Plasma and Brain in Senescence-Accelerated Aging and Alzheimer's disease Mouse Models
}

\author{
Shephali Bhatnagar, Gregory J. Weber, Vikranth Shetty, Viktoriya Shekhovtsova and Eugenia Wang
}

Advanced Genomic Technology, LLC, Louisville, Kentucky, 40202, USA

"Corresponding author: Eugenia Wang, 5100 US Highway 42, Suite 433, Louisville, Kentucky, 40241, Tel: (502) 228-5438; E-mail: ewangagt@gmail.com

Rec date: Jun 23, 2014, Acc date: Jul 26, 2014, Pub date: Aug 04, 2014

Copyright: (c) 2014 Bhatnagar S, et al. This is an open-access article distributed under the terms of the Creative Commons Attribution License, which permits unrestricted use, distribution, and reproduction in any medium, provided the original author and source are credited.

\begin{abstract}
Circulating microRNAs have recently emerged as powerful biomarkers because of their potential for monitoring changes in tissues such as the brain during aging. These microRNAs are found in many types of body fluids, and serve not only as systemic indicators of global and tissue-specific changes of gene expression, but also as functional mediators for cell-cell communication. In an effort to link changes in circulating microRNAs to changes in tissue, we used survival bleeding to perform comparative studies of three selected microRNAs, miR-34a, $-34 \mathrm{c}$, and $-181 \mathrm{~b}$, in plasma and brain tissue in two types of animal models, Alzheimer's Disease (AD) transgenic mutants and Senescence-Accelerated (SAMP8) mice. In this study, we show that expression of these microRNAs is altered in both AD and SAMP8 models, with comparable changes in expression of these specific microRNAs in both plasma and brain, and perhaps earlier in plasma. Our results demonstrate that the survival bleeding method allows longitudinal studies of changes in circulating microRNAs, which can be used as minimally invasive biomarkers of degenerative changes in mouse brain.
\end{abstract}

Keywords: Survival bleeding; Circulating microRNAs; SenescenceAccelerated Mouse; Alzheimer's disease

\section{Introduction}

Due to their stable presence in circulating blood, microRNAs are promising biological markers for the prognosis, diagnosis and treatment management of many diseases. The presence of circulating microRNAs in serum/plasma is largely due to two main factors: their presence in exosomal or multivesicular bodies, and their association with lipid particles. In both cases they are thought to function as complexes for cell-to-cell communication [1-3]. Circulating plasma miRNAs are emerging as practical diagnostic markers for detection of systemic changes, such as pregnancy trimester determination and central nervous system (CNS) disorders, including bipolar disorder, presymptomatic Huntington's disease, and Alzheimer's disease (AD) [4-7]. Neuronal communication at synapses is recently shown to involve exosomal transfer of proteins and RNA, including microRNAs [8]. Previous work in our laboratory has shown that human AD Peripheral Blood Mononuclear Cells (PBMC) and plasma exhibit patterns of miRNA expression differing from those of normal agematched controls [9-12]. Thus, circulating microRNA profiles are proving to be powerful blood-based biomarkers for brain aging and degeneration.

Apoptosis, or programmed cell death, is an important mechanism through which damaged or dying cells are removed from active tissue in adult organisms; it also plays a critical role in normal developmental processes [13]. In addition, there are clinical implications for studying the role of apoptosis in diseases, particularly neurodegenerative disorders, and the relationship between microRNA-regulated apoptotic signaling and the significance of the regulation/deregulation pertaining to the onset and progression of neurodegenerative diseases [13]. One particular group implicated in regulating genes directly involved in apoptotic signaling is the miR-34 family; increases in expression of miR-34a and miR-34c in AD plasma samples match corresponding decreases in BCL-2 and SIRT1 expression in the same samples [12]. This indicates that normal apoptotic and neuroprotective signaling pathways are compromised in $\mathrm{AD}$ patients, and the dysregulation of these microRNAs is critical to the pathogenesis of this disease. In addition, recent evidence has highlighted a role for miR-181b in targeted regulation of BCL-2 expression in pancreatic ductal adenocarcinoma, with increased expression leading to a decrease in BCL-2 expression [14]. Furthermore, miR-181b is implicated as a potential blood-based biomarker in leukemia, as well as regulating inflammatory signaling through the nuclear factor NF- $\kappa B$ pathway [15-17]. It is therefore plausible to suggest a role for miR-181b in Alzheimer's disease development and/or progression; increased plasma levels support miR181b as a blood-based biomarker for AD.

In addition to all the genetic risk factors discovered, age remains the best predictor for sporadic Alzheimer's disease, since most patients exhibit symptomatic onset after 65 years of age. Heritable AD polymorphisms largely involve three well-known genetic traits: 1) the apolipoprotein $\mathrm{E}$ (APOE) $\quad \varepsilon 4$ allele; 2) mutations involving accumulation of Amyloid Beta $(A \beta)$ peptide residues, with sequential cleavage of the Amyloid Precursor Protein (APP) by $\gamma$-secretase ( $\beta$-site APP cleaving enzyme 1, BACE1), involving the presenilin-1 complex; and 3) Tau phosphorylation [18-20]. Most AD transgenic mouse models are characterized by APP mutations known as the Swedish mutation (APPSWE) and Presenilin1 (PSEN1), along with hyperphosphorylated Tau mutations [21-23].Understandably, animal models such as APP and Tau mutants have been used to investigate microRNA changes in the brain, along with the altered pathology associated with beta-amyloid plaques and neurofibrillary tangle formation [24]. For example, in transgenic mice expressing mutated genes associated with Alzheimer's disease, such as double transgenic mutants carrying human APPSWE and PSEN1, both miR-34a \& -34c are increased in the hippocampus [25,26]. 
Citation: Bhatnagar S, Weber GJ, Shetty V, Shekhovtsova V, Wang E (2014) Compatible Changes of Lead microRNAs in Circulating Plasma and Brain in Senescence-Accelerated Aging and Alzheimer's disease Mouse Models. Aging Sci 2: 125. doi: $10.4172 / 2329-8847.1000125$

Page 2 of 8

The Senescence-Accelerated Mouse Prone 8 (SAMP8) strain developed by selective inbreeding of the AKR/J strain, exhibits an accelerated aging process and is a common animal model used in aging studies. In addition, SAMP8 mice are increasingly used for neurodegenerative studies, particularly in Alzheimer's disease; these mice develop pathologies similar to those observed in human Alzheimer's patients, including dementia, learning and memory deficits, $A \beta$ deposits, and altered expression of genes associated with the disease [27-29]. Conversely, the Senescence-Accelerated Mouse Resistant 1 (SAMR1) strain exhibits normal aging patterns, and is used as a complementary control strain to SAMP8 [28]. A recent study found accumulation of $A \beta$ in the hippocampus of SAMP8 mice, by observing positive staining with $A \beta 42$ and $A \beta 40$ antibodies [27]; this led to the conclusion that the SAMP8 mouse model is a viable animal system for studying accelerated aging and Alzheimer's disease.

This report presents data from survival bleeding, providing longitudinal blood samples from animals of three transgenic mouse strains carrying $\mathrm{AD}$ mutations and senescence-accelerated mice, as well as their corresponding controls, tracking the same individual mice during our study period of 12 months for the former, and 8 months for the latter. Comparison of levels of miR-34a, miR-34c and miR-181b between plasma and brain samples shows that significant differences between control and mutant strains can be seen earlier in circulating blood samples than in brain tissues of comparable age cohorts. Thus, our results suggest that changes in plasma microRNA levels, quantified in survival bled samples, may provide invaluable minimally invasive biomarkers for impending changes in mouse brain.

\section{Materials and Methods}

\section{Animal models and collection of blood}

Animals were purchased from either Taconic Laboratories, Inc. (Hudson, NY) or Harlan Laboratories, Inc. (UK), and used for the entire study. Transgenic mice carrying (1) a mutant form of amyloid precursor protein (APP) for A $\beta$ accumulation, APPSWE ("A"; 2789M) [21]; (2) a mutation for hyperphosphorylation of Tau, TAU ("T"; 2508-M) [23]; (3) both mutations, APPSWE-Tau (“AT”; 2469- RD1) [24]; (4) the control strain recommended for all three mutants ("WW"; 3273-M); (5) the senescence-prone inbred strain (SAMP8); and (6) the senescence-resistant inbred strain (SAMR1) [29]; were used in this study. All animals in this study were housed in the University of Louisville Animal Barrier Facility. $200 \mu \mathrm{l}$ of blood from mice at various ages was collected from the submandibular vein into BD microtainer tubes with EDTA (Fisher Scientific, cat\#02-669-38). EDTA was used as anticoagulant to obtain blood specimens to be processed for plasma samples as described below. In addition, mice not used for survival bleeding were sacrificed at corresponding time points; brain tissues were collected from these mice and flash-frozen in liquid nitrogen, for comparison with data obtained from the blood samples. All methods of housing and procedural work were approved by University of Louisville Institutional Animal Care and Use Committee protocol \#10130.

\section{Separation of blood into plasma fractions}

Plasma fractions were isolated from blood specimens by layering the blood onto Ficoll-Plaque Plus Solution (GE Healthcare,
Piscataway, NJ). Samples were then centrifuged for $30 \mathrm{~min}$ at $1,300 \mathrm{xg}$ at room temperature to separate the blood into plasma, peripheral blood mononuclear cells (PBMC), Ficoll-Plaque plus solution, and erythrocyte layers, from top to bottom respectively. The top layer containing plasma was collected and stored at $-80^{\circ} \mathrm{C}$ until further processing for RNA and protein isolation.

\section{RNA isolation and Quantitative PCR}

RNA fractions from both plasma and tissue samples were isolated as described previously [30]. The plasma was mixed with equal volume (1:1) of Trizol, followed by repetitive pipetting for 5 minutes. $30 \mathrm{mg}$ of tissue was homogenized in $1 \mathrm{ml}$ of Trizol reagent (Invitrogen). $200 \mu \mathrm{l}$ of chloroform per $1 \mathrm{ml}$ of Trizol was added to each plasma and tissue sample, followed by incubation at room temperature for 3 minutes. The mixture was centrifuged at $12,000 \mathrm{xg}$ for 15 minutes to separate the top RNA- containing aqueous phase, the middle DNA-containing layer (only in tissue samples), and the bottom protein-containing organic phase. In plasma samples, protein contamination was removed by adding equal volumes of Phenol: Chloroform: Isoamyl alcohol in the ratio of 25: 24: 1 and subsequently centrifuging again, as described above. The supernatant was collected and again mixed with equal volume of Phenol: Chloroform: Isoamyl alcohol in the ratio of 25: 24: 1. This protein decontamination step was repeated a second time to remove as much protein as possible. $500 \mu \mathrm{l}$ of Isopropyl alcohol was added to the supernatant collected from plasma samples, and the aqueous phase collected from tissues, to precipitate RNA. This was followed by washing in $75 \%$ ethanol. The precipitated plasma RNA, containing no $28 \mathrm{~S}$ or $18 \mathrm{~S}$ RNA species, was assessed for purity and integrity using a Nanodrop spectrophotometer (Thermo Fischer Scientific, Barrington, IL) and Agilent 2100 bioanalyzer (Agilent Technologies, Waldbronn, Germany).

RNA samples of optimal quality from three animals were selected from 5 mice of each cohort age group in each experiment performed in this study. Approximately $200 \mu \mathrm{g}$ of isolated RNA was used to generate cDNA, using the Taqman ${ }^{\bullet}$ MicroRNA Reverse Transcription Kit (Applied Biosystems, Carlsbad, CA), using specific miRNA stemloop primers for miR-34a, miR-34c, miR $181 \mathrm{~b}$ and miR-sno-202 (miR-34a:PN4427975-000426;miR-34c:PN4427975-000428;miR-181b: PN4427975-001098; miR-sno202:PN4427975-001232, Invitrogen), by enzyme MultiScribe Reverse Transcriptase (Applied Biosystems), in a GeneAmp PCR System 9700 (Applied Biosystems). Quantitative PCR (qPCR) reactions were set up using the Taqman Master Mix (Applied Biosystems). All reactions were performed in duplicate to reduce variation, and data were normalized by reference to the endogenous control (miR-sno-202) recommended by the manufacturer (Applied BioSystems) for mouse microRNA qPCR assays.

\section{Data and Statistical Analyses}

All analyses were conducted using MS Excel (Microsoft 2010) or SPSS software version 21 (IBM). The $\Delta \mathrm{Ct}$ value is calculated using the formula $(\Delta \mathrm{Ct}=\mathrm{Ct}$ of target gene-Ct of reference gene). $1 / \Delta \mathrm{Ct}$ values were used to represent qPCR results for expression levels of miR-34a, $\mathrm{miR}-34 \mathrm{c}$ and $\mathrm{miR}-181 \mathrm{~b}$ in various age groups. Average Ct values of each mouse strain for miR-sno-202 (reference gene) are graphically represented (Figure 1) using box plots; to show that there is no statistically significant difference between the groups. 
Citation: Bhatnagar S, Weber GJ, Shetty V, Shekhovtsova V, Wang E (2014) Compatible Changes of Lead microRNAs in Circulating Plasma and Brain in Senescence-Accelerated Aging and Alzheimer's disease Mouse Models. Aging Sci 2: 125. doi: $10.4172 / 2329-8847.1000125$

Page 3 of 8
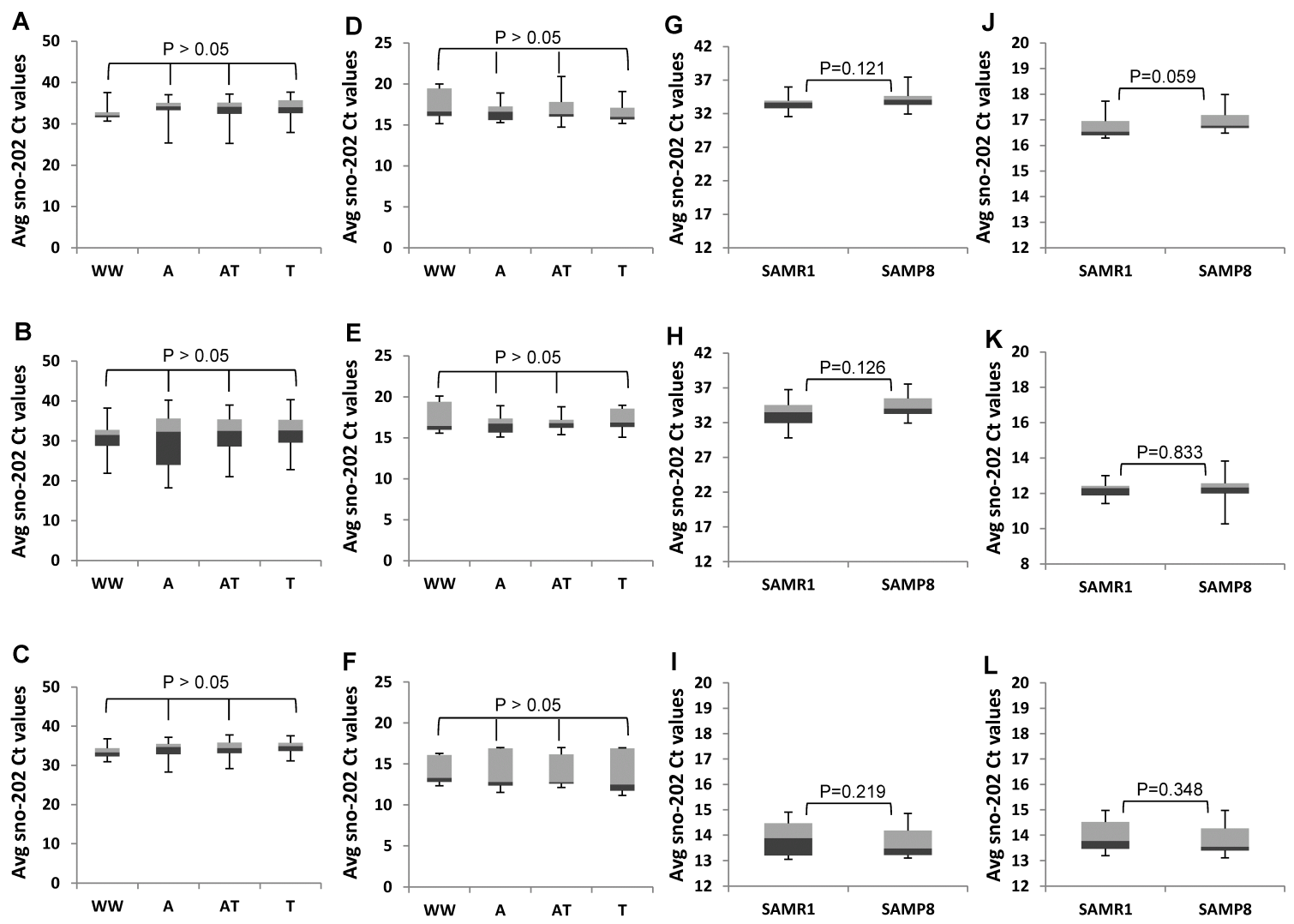

Figure 1: Average Ct values of reference target sno-202 used for qPCR in plasma and brain samples from AD transgenic, SAMP8 and SAMR1 mice. (A-C) Average Ct values in plasma for reference target sno-202, used for differential expression calculations in qPCR assays for miR-34a (A), miR-34c (B) and miR-181b (C). (D-F) Average Ct values in brain for reference target sno-202, used for differential expression calculations in qPCR assays for miR-34a (D), miR-34c (E) and miR-181b (F). The strains of mice used were: an APP mutant "A", double transgenic of both APP and Tau "AT", Tau mutant "T", and wild-type "WW". (G-I) Average Ct values in plasma for reference target sno-202, used for differential expression calculations in qPCR assays for miR-34a (G), miR-34c (H) and miR-181b (I). (J-L) Average Ct values in brain for reference target sno-202, used for differential expression calculations in qPCR assays for miR-34a (J), miR-34c (K) and miR-181b (L). The strains of mice used were SAMP8 and SAMR1. P-values represent the comparison of average Ct expression among the different strains used for the entire study. Error bars represent the range of $\mathrm{Ct}$ values within each strain for all time points tested in this study.

ANOVA followed by multiple comparison tests defined significant differences among more than two groups, and Student's t-test between two groups, with $\mathrm{p}$-values of $\mathrm{p}<0.05$ considered statistically significant $[31,32]$.

\section{Results}

\section{Levels of reference microRNA for qPCR assays in plasma and brain specimens}

Our animal protocol does not permit us to initiate survival bleeding before 4 months of age; therefore, blood samples were collected every month starting at 4 months until 12 months of age for the Alzheimer's disease mutant mice, and until 8 months of age for the senescenceaccelerated mouse mutant SAMP8 and its control SAMR1 strain. In general, from $200 \mu \mathrm{l}$ of blood collected per bleed per mouse, we were able to obtain sufficient quantities of the plasma fraction, which was further processed for isolation of RNA. We chose to use the Snord68 gene (referred to hereafter as sno-202), which yields a small nuclear RNA product upon transcription, as our control reference for qPCR assays. In an effort to determine the utility of this RNA as a suitable reference for our assays, the average $\mathrm{Ct}$ values for each animal strain tested at each time point, within each sample type (plasma or brain), were plotted to observe whether there was significant variation among the various animal strains used in this study. As seen in Figure 1, the range of average $\mathrm{Ct}$ values in plasma (Figure 1A-C) or brain (Figure 1D-F) samples for all time points tested did not significantly change among the different $\mathrm{AD}$ mutant strains over the course of the study. Similarly, the range of average Ct values in plasma (Figure 1G-I) or brain (Figure 1J-L) samples were not significantly different among the SAMP8 and SAMR1 mice when considering all the time points tested in this study. Thus, the consistent average expression of sno-202 makes it a suitable reference for normalizing differential expression in our qPCR assays. 
Citation: Bhatnagar S, Weber GJ, Shetty V, Shekhovtsova V, Wang E (2014) Compatible Changes of Lead microRNAs in Circulating Plasma and Brain in Senescence-Accelerated Aging and Alzheimer's disease Mouse Models. Aging Sci 2: 125. doi: $10.4172 / 2329-8847.1000125$

Page 4 of 8

\begin{tabular}{|l|l|l|l|l|l|l|l|l|l|l|}
\hline Target & Comparison & $\mathbf{4 m}$ & $\mathbf{5 m}$ & $\mathbf{6 m}$ & $\mathbf{7 m}$ & $8 \mathrm{~m}$ & $9 \mathrm{~m}$ & $10 \mathrm{~m}$ & $11 \mathrm{~m}$ & $12 \mathrm{~m}$ \\
\hline \multirow{3}{*}{ miR-34a } & WW vs. A & $0.01^{*}$ & $<0.001^{*}$ & 0.186 & 0.077 & $0.033^{*}$ & 0.353 & 0.246 & 0.55 & 0.546 \\
\cline { 2 - 10 } & WW vs. AT & $0.023^{*}$ & $0.008^{*}$ & 0.134 & $0.014^{*}$ & $<0.001^{*}$ & $0.005^{*}$ & $<0.001^{*}$ & $0.016^{*}$ & $0.010^{*}$ \\
\cline { 2 - 10 } & WW vs. T & $<0.001^{*}$ & $<0.001^{*}$ & 0.083 & $0.021^{*}$ & $<0.001^{*}$ & $0.004^{*}$ & $<0.001^{*}$ & $0.014^{*}$ & $0.005^{*}$ \\
\hline \multirow{3}{*}{ miR-34c } & WW vs. A & 0.571 & 0.083 & 0.425 & $<0.001^{*}$ & $0.007^{*}$ & $<0.001^{*}$ & $0.008^{*}$ & 0.071 & $0.004^{*}$ \\
\cline { 2 - 10 } & WW vs. AT & 0.574 & 0.781 & 0.132 & $<0.001^{*}$ & $<0.001^{*}$ & $<0.001^{*}$ & $<0.001^{*}$ & $0.009^{*}$ & $<0.001^{*}$ \\
\cline { 2 - 10 } & WW vs. T & $0.020^{*}$ & 0.688 & $0.002^{*}$ & $<0.001^{*}$ & $<0.001^{*}$ & $<0.001^{*}$ & $<0.001^{*}$ & $0.005^{*}$ & $<0.001^{*}$ \\
\hline \multirow{3}{*}{ miR-181b } & WW vs. A & 0.287 & 0.061 & 0.057 & 0.158 & 0.16 & 0.062 & 0.109 & $0.024^{*}$ & $0.021^{*}$ \\
\cline { 2 - 10 } & WW vs. AT & 0.064 & 0.077 & 0.062 & 0.104 & 0.097 & $0.042^{*}$ & 0.086 & $0.011^{*}$ & $0.010^{*}$ \\
\cline { 2 - 9 } & WW vs. T & 0.15 & $0.003^{*}$ & $0.009^{*}$ & 0.053 & 0.088 & $0.025^{*}$ & $0.033^{*}$ & $0.002^{*}$ & $0.004^{*}$ \\
\hline
\end{tabular}

Table 1: p-values of miR-34a, miR-34c, and miR-181b levels in Alzheimer's disease (AD) transgenic mutants in plasma: APP mutant "A”, double transgenic of both APP and Tau "AT", Tau mutant "T", and wild-type "WW". Significance is defined as a p-value of $p<0.05$, and is denoted by $\left({ }^{*}\right)$.

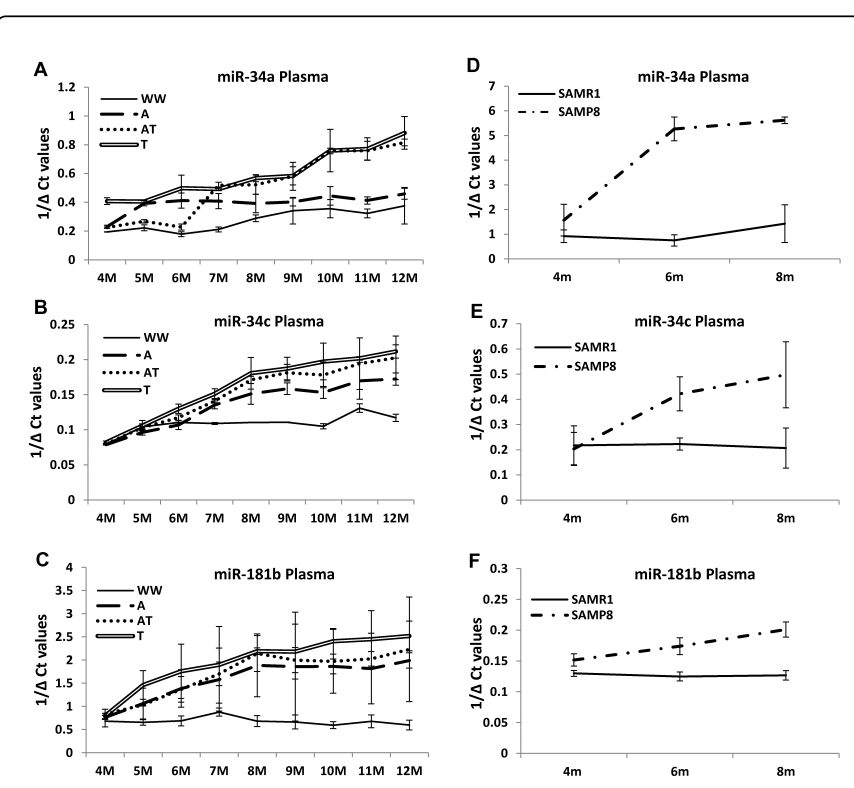

Figure 2: Levels of miR-34a, miR-34c, and miR-181b in plasma samples in AD transgenic, SAMR1 and SAMP8 mice. (A-C) Changes in expression of miR-34a (A), miR-34c (B) and miR-181b (C) in APP mutant "A", double transgenic of both APP and Tau "AT", Tau mutant "T" and "WW" control mice at ages from 4 to 12 months. (D-F) Expression of transcript levels of miR-34a (D), miR-34c (E) and miR-181b (F) in SAMP8 and SAMR1 (control) mice aged 4 to 8 months, measured by qPCR assay. All target microRNAs assayed by qPCR are expressed as $1 / \Delta C t$ with $n=3$ for each strain. Error bars are expressed as $\pm \mathrm{SD}$. Levels of miR-34a, $-34 \mathrm{c}$ and $-181 \mathrm{~b}$ in circulating plasma of
survival-bled samples from young to old age in three

\section{Alzheimer's disease (AD) transgenic mouse mutant strains and the senescence-accelerated mouse (SAMP8) model}

Due to the limited quantities of RNA acquired from the survival bled specimens, we opted to focus on three specific microRNAs, miR-34a, -34c, and -181b. We have previously shown an increase in expression of the first two microRNAs in peripheral blood mononuclear cells and plasma specimens of Alzheimer's disease patients $[9,12]$.

As shown in Figure 2A and Table 1, as early as the beginning of our study, i.e. 4 months of age, the Tau transgenic mice show significantly higher levels of miR-34a compared with wild-type controls $(\mathrm{p}<0.001)$, while in the APP strain miR-34a is moderately increased at 4 months of age $(p=0.010)$, and plateaus afterward. The increase of this microRNA level in the double transgenic strain does not become obvious until 7 months old; this trend continues afterwards, showing modest increase in expression (Figure 2A).

The pattern of increased expression is also observed with the sister microRNA, miR-34c. However, the increase in the three mutant strains does not differ from the wild-type control mice until after 6 months of age. The increase trend continues from 7 months of age until the end of our study period, i.e. the 12 -month time point, while miR-34c levels in control mice remain at the 6-month level through the remaining time points until 12 months of age (Figure $2 \mathrm{~B}$ and Table $1)$.

For miR-181b, as early as 5 months of age, quantitative PCR (qPCR) analyses of plasma levels reveal a significant increase in the Tau mutant $(\mathrm{p}=0.003)$, and modest increases in the APP and double mutants, compared with wild-type controls (Figure 2C, Table 1). The trend of increased expression of miR-181b continues in the Tau mutant mice from 6 months until 9-12 months of age. A similar increase is also observed in the double transgenic and APP mutants, but at somewhat lower levels (Figure 2C and Table 1).

Quantitative PCR analysis of transcript levels of miR-34a, -34c, and $-181 \mathrm{~b}$ in SAMP8 mice were compared to SAMR1 mice at several time points. There is a significantly higher level of expression of miR-34a $(\mathrm{p}=0.027)$ at 4 months of age in SAMP8 mice (Figure 2D and Table 2). 
Citation: Bhatnagar S, Weber GJ, Shetty V, Shekhovtsova V, Wang E (2014) Compatible Changes of Lead microRNAs in Circulating Plasma and Brain in Senescence-Accelerated Aging and Alzheimer's disease Mouse Models. Aging Sci 2: 125. doi: $10.4172 / 2329-8847.1000125$

Page 5 of 8

\begin{tabular}{|l|l|l|l|l|}
\hline Target & Comparison & $\mathbf{4 m}$ & $\mathbf{6 m}$ & $8 \mathrm{~m}$ \\
\hline miR-34a & SAMR1 vs. SAMP8 & $0.027^{*}$ & $0.035^{*}$ & $<0.001^{*}$ \\
\hline miR-34c & SAMR1 vs. SAMP8 & 0.772 & $0.027^{*}$ & $0.013^{*}$ \\
\hline miR-181b & SAMR1 vs. SAMP8 & $0.016^{*}$ & $0.013^{*}$ & $<0.001^{*}$ \\
\hline
\end{tabular}

\begin{tabular}{|l|l|l|l|l|}
\hline & WW vs. AT & 0.988 & 0.062 & $0.023^{*}$ \\
\cline { 2 - 5 } & WW vs. T & 0.879 & 0.057 & $0.022^{*}$ \\
\hline
\end{tabular}

Table 3: p-values of miR-34a, miR-34c, and miR-181b levels in Alzheimer's disease (AD) transgenic mouse mutants in brain: APP mutant "A", double transgenic of both APP and Tau "AT", Tau

Table 2: p-values of miR-34a, miR-34c, and miR-181b levels in senescence-accelerated mouse (SAMP8) and its control strain (SAMR1) mice in plasma. Significance is defined as a p-value of $\mathrm{p}<0.05$, and is denoted by $\left(^{*}\right)$.

In addition, $\mathrm{qPCR}$ results reveal a continued increase in expression in plasma samples at 6 months of age, with plasma miR-34a rising slightly until 8 months of age (Figure 2D). In the case of miR-34c, we do not observe the early higher level of expression, but a significant increase in expression in plasma at 6 months of age $(p=0.027)$, with levels continuing to increase in plasma at 8 months of age in SAMP8 compared to SAMR1 mice (Figure 2E and Table 2). Transcript levels of miR-181b show a small but significant increase $(\mathrm{p}=0.016)$ in expression in SAMP8 mice at 4 months in plasma compared to SAMR1 mice (Figure $2 \mathrm{~F}$ and Table 2). At 6 months of age, there is considerably higher expression of miR-181b in plasma; the trend of increasing levels of miR-181b in plasma is observed all the way to 8 months (Figure 2F and Table 2).

\section{Levels of miR-34a, $-34 c$ and -181b in brain samples of Alzheimer's disease (AD) transgenic mutants and senescence-accelerated aging mouse models}

Since the earliest positive immunogenic amyloid-like structures are detected at the age of 6-7 months in the brain of APP transgenecarrying mutants [33-35], we selected three time points, 4, 7.5 and 12 months, representing the earliest plasma specimen collection, the stage when amyloid-like structures are detected, and old age cohorts respectively. Quantitative PCR analyses of miR-34a expression in brain samples of Tau and double transgenic APP-Tau mutants show a slight increase at the age of 7.5 months, with the APP mutants showing the greatest increase (Figure 3A). By 12 months, all three transgenic mutants show robustly increased miR-34a expression compared with wild-type controls. The increased miR-34c level in brain is not as dramatic as miR-34a at 7.5 months; nevertheless, a significant increase is seen in the mutants carrying either single Tau or double transgenic of both Tau and APP at 7.5 months, with all three transgenic mouse strains showing significant differences compared with wild-type mice by 12 months for this microRNA, with increases similar to that of its sister microRNA (Figure 3B and Table 3).

\begin{tabular}{|l|l|l|l|l|}
\hline Target & Comparison & $\mathbf{4 m}$ & $7.5 \mathrm{~m}$ & $12 \mathrm{~m}$ \\
\hline \multirow{3}{*}{ miR-34a } & WW vs. A & 0.781 & 0.159 & $0.001^{*}$ \\
\cline { 2 - 5 } & WW vs. AT & 0.491 & 0.733 & $<0.001^{*}$ \\
\cline { 2 - 5 } & WW vs. T & 0.734 & 0.45 & $0.002^{*}$ \\
\hline \multirow{3}{*}{ miR-34c } & WW vs. A & 0.91 & 0.061 & $<0.001^{*}$ \\
\cline { 2 - 5 } & WW vs. AT & 0.805 & $0.004^{*}$ & $<0.001^{*}$ \\
\cline { 2 - 5 } & WW vs. T & 0.739 & $0.008^{*}$ & $<0.001^{*}$ \\
\hline \multirow{2}{*}{ miR-181b } & WW vs. A & 0.983 & $0.045^{*}$ & $0.033^{*}$ \\
\hline
\end{tabular}
mutant "T", and wild-type "WW". Significance is defined as a p-value of $\mathrm{p}<0.05$, and is denoted by $\left(^{*}\right)$.

Transcript levels of miR-181b increase significantly at 7.5 months in the APP-transgenic mouse mutants compared to control mice, and by 12 months, significant increases are clear in all three transgenic mice (APP, APP-Tau, and Tau) (Figure 3C and Table 3).
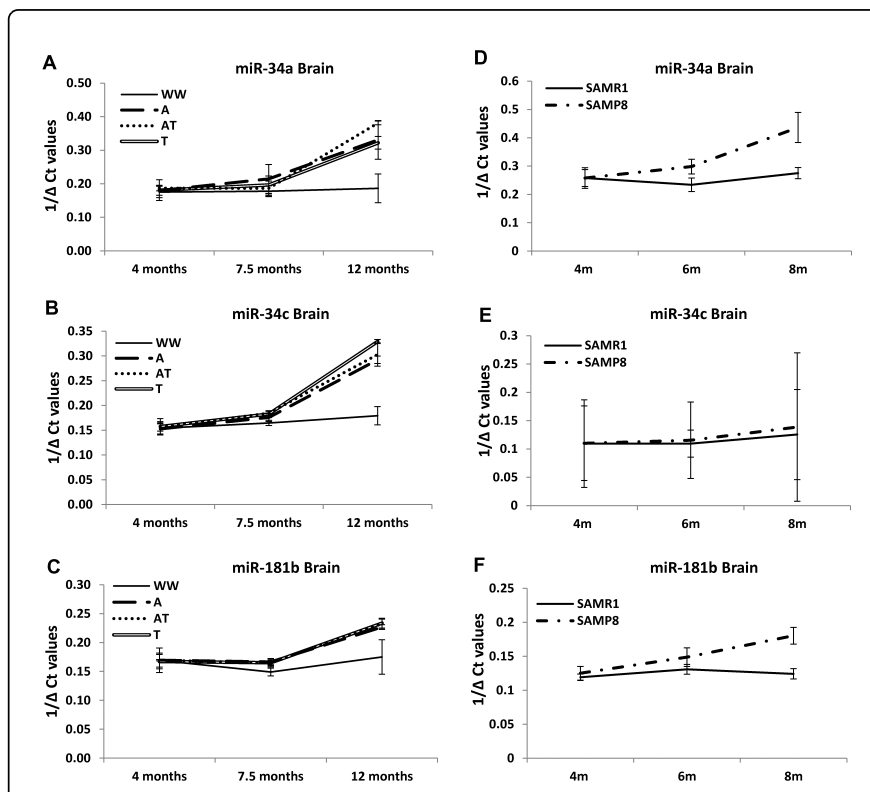

Figure 3: Levels of miR-34a, miR34c, and miR-181b in brain samples of AD transgenic, SAMR1 and SAMP8 mice. (A-C) Expression levels of miR-34a (A), miR-34c (B) and miR-181b (C) in brain tissues from APP mutant "A", double transgenic mutant carrying both APP and Tau mutation "AT", Tau mutation "T" mice, and wild-type controls for all three mutants "WW" at ages ranging from 4 to 12 months. (D-F) Expression levels of miR-34a (D), miR-34c (E), and miR-181b (F) in SAMP8 and SAMR1 (control) mice aged 4 to 8 months in brain tissue, measured by qPCR. All target microRNAs assayed by qPCR are expressed as $1 /$ $\Delta \mathrm{Ct}$, with $\mathrm{n}=3$ for each strain. Error bars are expressed as $\pm \mathrm{SD}$.

We next investigated the expression levels of miR-34a, $-34 c$, and $-181 \mathrm{~b}$ in the brain tissue of SAMP8 and SAMR1 mice. miR-34a expression levels steadily increase from 4 months all the way to 8 months of age, with a stronger increase from 6 to 8 months (Figure $3 \mathrm{D})$. However, there is no significant difference in miR-34c levels in the brain at any time point observed (Figure 3E and Table 4). The third microRNA studied in the brain is miR-181b, with a slight increase in SAMP8 mouse brain tissue starting at 4 months; however this increase was not statistically significant until 8 months (Figure $3 \mathrm{~F}$ and Table 4).

\begin{tabular}{|l|l|l|l|l|}
\hline Target & Comparison & $4 \mathrm{~m}$ & $6 \mathrm{~m}$ & $8 \mathrm{~m}$ \\
\hline
\end{tabular}


Citation: Bhatnagar S, Weber GJ, Shetty V, Shekhovtsova V, Wang E (2014) Compatible Changes of Lead microRNAs in Circulating Plasma and Brain in Senescence-Accelerated Aging and Alzheimer's disease Mouse Models. Aging Sci 2: 125. doi: $10.4172 / 2329-8847.1000125$

Page 6 of 8

\begin{tabular}{|l|l|l|l|l|}
\hline miR-34a & SAMR1 vs. SAMP8 & 0.996 & $0.035^{*}$ & $0.005^{*}$ \\
\hline miR-34c & SAMR1 vs. SAMP8 & 0.821 & 0.271 & 0.128 \\
\hline miR-181b & SAMR1 vs. SAMP8 & 0.362 & 0.053 & $0.011^{*}$ \\
\hline
\end{tabular}

expression, and indicate links between certain types of disease and types of microRNA exhibiting altered expression. Our selection of the three microRNAs, miR-34a, $-34 c$ and $-181 b$, is based on previous evidence that miR-34a and $-181 \mathrm{~b}$ are two leading microRNAs increased in abundance in Peripheral Blood Mononuclear Cells (PBMC) of sporadic AD victims [9]. In addition, miR-34c is also

Table 4: $p$-values of miR-34a, miR-34c, and miR-181b levels in brain of senescence-accelerated mouse (SAMP8) and its control strain (SAMR1). Significance is defined as a $\mathrm{p}$-value of $\mathrm{p}<0.05$, and is denoted by $\left(^{*}\right)$.

\section{Discussion}

Survival bleeding for blood microRNA biomarkers allows analyzing the same individual mice at multiple time points over the course of the study period. This provides true temporal kinetic evaluation of changes within individual mice, without the inevitable inter-animal variation associated with comparing blood samples collected through sacrifice of different cohorts at scheduled intervals over a longitudinal study. Previous results from our laboratory, reporting systemic versus tissue- specific changes in key miRNAs in brain and liver of aged C57BL/6 mice, suggest that a decline in health with age may be stimulated by a systemic deterioration of signaling in diverse tissues, impacted by up-regulated shared key microRNAs (e.g. miR-34a), and individual tissue functional decline regulated by tissue-specific miRs, such as miR-669c, -712, -93, and -214 (in liver), and miR-22, -101a, -720 , and -721 (in brain) [36-38]. Among these changes, miR-34a is also increased in mouse plasma specimens during aging [30]. Traditionally, animal studies involving changes in the brain during aging require large animal colonies, with experimental designs sacrificing at least three animals each from several age groups during the lifespan to evaluate effects of aging. By this experimental design, the earliest amyloid-like structures discernible by specific antibody detection are reported at 6-7 months [26,33-35]. This approach incurs significant costs of maintaining large animal colonies, and more importantly introduces unavoidable inter-animal variation within cohorts used at different time points. Our adopted survival bleeding method via submandibular vein [39] allows us to obtain serial blood samples from surviving mice, avoiding the more invasive use of the jugular vein or cardiac puncture upon euthanasia. Here, we employ age-accelerated mouse model SAMP8 and SAMR1 to evaluate similarities of $\mathrm{AD}$ progression to the aging process, as well as three $\mathrm{AD}$ transgenic mouse models widely used in studying the effects of mutations in APP, Tau, and both genes [24].

MicroRNAs are small ( $22 \mathrm{nt})$ non-protein-coding RNAs, which bind with partial complementarity to messenger RNA (mRNA) sequences, mainly in the $3^{\prime}$ untranslated region (3' UTR), leading to degradation or translational repression of the mRNA template. This, in turn, results in down- regulation of protein output of the targeted mRNA $[40,41]$. These small RNAs are highly stable in body fluids such as plasma and cerebrospinal fluid (CSF) [42], and are transported in exosomes or liposomes [43]. A number of microRNAs, such as miR-34c, miR-128a, miR-146a, miR-181b, and miR-34a are dysregulated in $\mathrm{AD}$ patients and animal models of $\mathrm{AD}[10,44,45]$.

An interesting observation pointed out in the literature is that while several microRNAs, such as miR-34c, are increased in expression in $\mathrm{AD}$ models, their expression is substantially decreased in other diseases, including several types of cancer [46]. The timely and specific balance of the expression of these microRNAs may lend insight into the various disease pathways that can arise from their altered increased in the hippocampus of $\mathrm{AD}$ transgenic mice and $\mathrm{AD}$ plasma $[12,26]$. Moreover, it is noteworthy that this latter study also found miR-34c to be highly enriched in the hippocampus, and linked with other learning-associated genes [26]. Furthermore, miR-34a is increased in the brains of AD Tg mice [20], and in aged C57-black mouse brain and circulating plasma [37,38]; this increased expression of miR-34a may be attenuated by caloric restriction [37].

We also investigated the potential of early detection of circulating microRNAs in an age-accelerated mouse model, SAMP8. The levels of miR-34a and miR-181b show statistically significant increases as early as 4 months in plasma of SAMP8 mice; and by six months, all three microRNAs are significantly higher than in wild-type mice (Table 2). It is intriguing that the levels of some of these microRNAs, especially miR-181b, are significantly increased in plasma as early as 4-5 months in both the AD mutant and SAMP8 mouse models (Figure 2). While $\mathrm{miR}-34 \mathrm{a}$ and miR-34c expression levels are not as robustly increased as miR-181b in these mice, an increase in plasma levels of these microRNAs is evident well before an appreciable increase is observed in the brain. Clearly, inter- mutant differences for three individual microRNAs' rise in plasma are seen by the comparative analysis of $\mathrm{p}$ values, as shown in Table 1. Nevertheless, our results provide initial evidence that the microRNAs included in this study have the potential to be used as powerful plasma-based diagnostic tools to monitor brain-related changes in mouse mutants carrying transgenes associated with the plaques and tangles observed in Alzheimer's disease brain.

Studies in the past have suggested that oxidative damage is associated with early events in the developmental pathology of $\mathrm{AD}$ [47-50]. In the present study, the levels of these three microRNAs increase earlier in plasma than in brain tissue in older mice of the AD mutants and the SAMP8 strain. These results lead us to suggest that increased levels of these microRNAs may be among the important mechanisms leading to overall systemic weakening of stress defense and cell survival in these mutant strains. Life-long cumulative oxidative stress may activate the expression of some of these microRNAs, with a progressive trend during aging [51]. A plethora of literature suggests that oxidative stress is enhanced in multiple tissues, including brain, of old mice. We believe that increased levels of miR-34a, miR-34c and miR-181b may be among the potential mechanisms for age-related increase in oxidative stress, not only during normal aging but also possibly during the accelerated aging observed in the SAMP8 strain as well as in AD mutant strains.

In conclusion, our results suggest that the survival bleeding method used to obtain plasma samples provides ready access to early temporal changes in circulating blood biomarkers. The levels of these three microRNAs increasing in plasma samples at an early age suggest that circulating blood biomarkers represent a powerful tool for minimally invasive detection of changes in brain in animal models, as reported here in accelerated aging and $\mathrm{AD}$ mutant strains. 
Citation: Bhatnagar S, Weber GJ, Shetty V, Shekhovtsova V, Wang E (2014) Compatible Changes of Lead microRNAs in Circulating Plasma and Brain in Senescence-Accelerated Aging and Alzheimer's disease Mouse Models. Aging Sci 2: 125. doi: $10.4172 / 2329-8847.1000125$

Page 7 of 8

\section{Acknowledgement}

The authors wish to thank Mr. Alan N. Bloch for proof-reading the manuscript, and Mr. Nathan Shelburne for performing the bioinformatic analysis. This work was supported by grants from the Kentucky Science and Technology Corporation (KSTC) to Advanced Genomic Technology, LLC.

\section{Disclosure Statement}

Eugenia Wang is on entrepreneurial leave from the University of Louisville, with $51 \%$ of her effort committed to Advanced Genomic Technology, LLC, a start-up biotech company in Louisville, Kentucky; her other $49 \%$ is at the University of Louisville School of Medicine, as the Gheens Endowed Chair on Aging, and Professor of Biochemistry and Molecular Biology. Eugenia Wang, Shephali Bhatnagar, Vikranth Shetty, Viktoriya Shekhovtsova and Gregory Weber are employees of Advanced Genomic Technology. The animals used in this study were purchased from Taconic Laboratories, Inc. or Harlan Laboratories, Inc. and were used for research purposes only.

\section{References}

1. Rykova EY, Morozkin ES, Ponomaryova AA, Loseva EM, Zaporozhchenko IA, et al. (2012) Cell-free and cell-bound circulating nucleic acid complexes: mechanisms of generation, concentration and content. Expert Opin Biol Ther 12 Suppl 1: S141-153.

2. Pritchard CC, Kroh E, Wood B, Arroyo JD, Dougherty KJ, et al. (2012) Blood cell origin of circulating microRNAs: a cautionary note for cancer biomarker studies. Cancer Prev Res (Phila) 5: 492-497.

3. Kosaka N, Iguchi H, Ochiya T (2010) Circulating microRNA in body fluid: a new potential biomarker for cancer diagnosis and prognosis. Cancer Sci 101: 2087-2092.

4. Kotlabova K, Doucha J, Hromadnikova I (2011) Placental-specific microRNA in maternal circulation--identification of appropriate pregnancy-associated microRNAs with diagnostic potential. J Reprod Immunol 89: 185-191.

5. Suárez-Gómez M, Alejandre-Durán E, Ruiz-Rubio M (2011) [MicroRNAs in bipolar disorder: diagnostic and therapeutic applications]. Rev Neurol 53: 91-98.

6. Gaughwin PM, Ciesla M, Lahiri N, Tabrizi SJ, Brundin P, et al. (2011) Hsa-miR-34b is a plasma-stable microRNA that is elevated in premanifest Huntington's disease. Hum Mol Genet 20: 2225-2237.

7. Geekiyanage H, Jicha GA, Nelson PT, Chan C (2012) Blood serum miRNA: non-invasive biomarkers for Alzheimer's disease. Exp Neurol 235: 491-496.

8. Smalheiser NR (2007) Exosomal transfer of proteins and RNAs at synapses in the nervous system. Biol Direct 2: 35.

9. Schipper HM, Maes OC, Chertkow HM, Wang E (2007) MicroRNA expression in Alzheimer blood mononuclear cells. Gene Regul Syst Bio 1: 263-274.

10. Maes OC, Chertkow HM, Wang E, Schipper HM (2009) MicroRNA: Implications for Alzheimer Disease and other Human CNS Disorders. Curr Genomics 10: 154-168.

11. Maes OC, Schipper HM, Chertkow HM, Wang E (2009) Methodology for discovery of Alzheimer's disease blood-based biomarkers. J Gerontol A Biol Sci Med Sci 64: 636-645.

12. Bhatnagar S, Chertkow H, Schipper HM, Yuan Z, Shetty V, et al. (2014) Increased microRNA-34c abundance in Alzheimer's disease circulating blood plasma. Front Mol Neurosci 7: 2.

13. Mattson MP (2006) Neuronal life-and-death signaling, apoptosis, and neurodegenerative disorders. Antioxid Redox Signal 8: 1997-2006.
14. Cai B, An Y, Lv N, Chen J, Tu M, et al. (2013) miRNA-181b increases the sensitivity of pancreatic ductal adenocarcinoma cells to gemcitabine in vitro and in nude mice by targeting BCL-2. Oncol Rep 29: 1769-1776.

15. Visone R, Veronese A, Rassenti LZ, Balatti V, Pearl DK, et al. (2011) miR-181b is a biomarker of disease progression in chronic lymphocytic leukemia. Blood 118: 3072-3079.

16. Zhi F, Cao X, Xie X, Wang B, Dong W, et al. (2013) Identification of circulating microRNAs as potential biomarkers for detecting acute myeloid leukemia. PLoS One 8: e56718.

17. Sun X, He S, Wara AK, Icli B, Shvartz E, et al. (2014) Systemic delivery of microRNA-181b inhibits nuclear factor- $\kappa$ B activation, vascular inflammation, and atherosclerosis in apolipoprotein E-deficient mice. Circ Res 114: 32-40.

18. Bertram L, Lill CM, Tanzi RE (2010) The genetics of Alzheimer disease: back to the future. Neuron 68: 270-281.

19. Martin L, Latypova X, Terro F (2011) Post-translational modifications of tau protein: implications for Alzheimer's disease. Neurochem Int 58: 458-471.

20. Alonso AC, Li B, Grundke-Iqbal I, Iqbal K (2008) Mechanism of tauinduced neurodegeneration in Alzheimer disease and related tauopathies. Curr Alzheimer Res 5: 375-384.

21. Hsiao K, Chapman P, Nilsen S, Eckman C, Harigaya Y, et al. (1996) Correlative memory deficits, $A ß$ elevation, and amyloid plaques in transgenic mice. Science. 274:99-102.

22. Lewis J, McGowan E, Rockwood J, Melrose H, Nacharaju P, et al. (2000) Neurofibrillary tangles, amyotrophy and progressive motor disturbance in mice expressing mutant (P301L) tau protein. Nat Genet 25: 402-405.

23. Lewis J, Dickson DW, Lin WL, Chisholm L, Corral A, et al. (2001) Enhanced neurofibrillary degeneration in transgenic mice expressing mutant tau and APP. Science 293: 1487-1491.

24. Woodruff-Pak DS (2008) Animal models of Alzheimer's disease: therapeutic implications. J Alzheimers Dis 15: 507-521.

25. Wang X, Liu P, Zhu H, Xu Y, Ma C, et al. (2009) miR-34a, a microRNA up-regulated in a double transgenic mouse model of Alzheimer's disease, inhibits bcl2 translation. Brain Res Bull 80: 268-273.

26. Zovoilis A, Agbemenyah HY, Agis-Balboa RC, Stilling RM, Edbauer D, et al. (2011) microRNA-34c is a novel target to treat dementias. EMBO J 30: 4299-4308.

27. Del Valle J, Duran-Vilaregut J, Manich G, Casadesús G, Smith MA, et al. (2010) Early amyloid accumulation in the hippocampus of SAMP8 mice. J Alzheimers Dis 19: 1303-1315.

28. Spangler EL, Patel N, Speer D, Hyman M, Hengemihle J, et al. (2002) Passive avoidance and complex maze learning in the senescence accelerated mouse (SAM): age and strain comparisons of SAM P8 and R1. J Gerontol A Biol Sci Med Sci 57: B61-68.

29. Takeda $\mathrm{T}$ (2009) Senescence-accelerated mouse (SAM) with special references to neurodegeneration models, SAMP8 and SAMP10 mice. Neurochem Res 34: 639-659.

30. Li X, Khanna A, Li N, Wang E (2011) Circulatory miR34a as an RNAbased, noninvasive biomarker for brain aging. Aging (Albany NY) 3: 985-1002.

31. Sawyer SF (2009) Analysis of Variance: The fundamental concepts. J Manual \& Manipulative Therapy. 17:27E-38E.

32. Rojewski JW, Lee IH, Geminci S. (2012) Use of t-test and ANOVA in Career-Technical Education Research. CTER. 37:263-275.

33. Wilcock DM, Colton CA (2008) Anti-amyloid-beta immunotherapy in Alzheimer's disease: relevance of transgenic mouse studies to clinical trials. J Alzheimers Dis 15: 555-569.

34. Green KN, Steffan JS, Martinez-Coria H, Sun X, Schreiber SS, et al. (2008) Nicotinamide restores cognition in Alzheimer's disease transgenic mice via a mechanism involving sirtuin inhibition and selective reduction of Thr231-phosphotau. J Neurosci 28: 11500-11510.

35. Qin W, Yang T, Ho L, Zhao Z, Wang J, et al. (2006) Neuronal SIRT1 activation as a novel mechanism underlying the prevention of Alzheimer 
Citation: Bhatnagar S, Weber GJ, Shetty V, Shekhovtsova V, Wang E (2014) Compatible Changes of Lead microRNAs in Circulating Plasma and Brain in Senescence-Accelerated Aging and Alzheimer's disease Mouse Models. Aging Sci 2: 125. doi: $10.4172 / 2329-8847.1000125$

Page 8 of 8

disease amyloid neuropathology by calorie restriction. J Biol Chem 281: $21745-21754$

36. Li N, Bates DJ, An J, Terry DA, Wang E (2011) Up-regulation of key microRNAs, and inverse down-regulation of their predicted oxidative phosphorylation target genes, during aging in mouse brain. Neurobiol Aging 32: 944-955.

37. Khanna A, Muthusamy S, Liang R, Sarojini H, Wang E (2011) Gain of survival signaling by down-regulation of three key miRNAs in brain of calorie-restricted mice. Aging (Albany NY) 3: 223-236.

38. Li N, Muthusamy S, Liang R, Sarojini H, Wang E (2011) Increased expression of miR-34a and miR-93 in rat liver during aging, and their impact on the expression of Mgst1 and Sirt1. Mech Ageing Dev 132: 75-85.

39. Golde WT, Gollobin P, Rodriguez LL (2005) A rapid, simple, and humane method for submandibular bleeding of mice using a lancet. Lab Anim (NY) 34: 39-43.

40. Treiber T, Treiber N, Meister G (2012) Regulation of microRNA biogenesis and function. Thromb Haemost 107: 605-610.

41. Abe M, Bonini NM (2013) MicroRNAs and neurodegeneration: role and impact. Trends Cell Biol 23: 30-36.

42. Vickers KC, Palmisano BT, Shoucri BM, Shamburek RD, Remaley AT (2011) MicroRNAs are transported in plasma and delivered to recipient cells by high-density lipoproteins. Nat Cell Biol 13: 423-433.

43. Hu G, Drescher KM, Chen XM (2012) Exosomal miRNAs: Biological Properties and Therapeutic Potential. Front Genet 3: 56.
44. Müller M, Kuiperij HB, Claassen JA, Küsters B, Verbeek MM (2014) MicroRNAs in Alzheimer's disease: differential expression in hippocampus and cell-free cerebrospinal fluid. Neurobiol Aging 35: 152-158.

45. Wang X, Liu P, Zhu H, Xu Y, Ma C, et al. (2009) miR-34a, a microRNA up-regulated in a double transgenic mouse model of Alzheimer's disease, inhibits bcl2 translation. Brain Res Bull 80: 268-273.

46. Holohan KN, Lahiri DK, Schneider BP, Foroud T, Saykin AJ (2013) Functional microRNAs in Alzheimer's disease and cancer: differential regulation of common mechanisms and pathways. Front Genet 3: 323.

47. Holohan KN, Lahiri DK, Schneider BP, Foroud T, Saykin AJ (2013) Functional microRNAs in Alzheimer's disease and cancer: differential regulation of common mechanisms and pathways. Front Genet 3: 323.

48. Su B, Wang X, Nunomura A, Moreira PI, Lee HG, et al. (2008) Oxidative stress signaling in Alzheimer's disease. Curr Alzheimer Res 5: 525-532.

49. Butterfield DA, Drake J, Pocernich C, Castegna A (2001) Evidence of oxidative damage in Alzheimer's disease brain: central role for amyloid beta-peptide. Trends Mol Med 7: 548-554.

50. Clotman F, Jacquemin P, Plumb-Rudewiez N, Pierreux CE, Van der Smissen P, et al. (2005) Control of liver cell fate decision by a gradient of TGF beta signaling modulated by Onecut transcription factors. Genes Dev 19: 1849-1854.

51. Goodall EF, Heath PR, Bandmann O, Kirby J, Shaw PJ (2013) Neuronal dark matter: the emerging role of microRNAs in neurodegeneration. Front Cell Neurosci 7: 178 